\title{
The role of learning in remembered duration
}

\author{
MARILYN G. BOLTZ, CARA KUPPERMAN, and JESSICA DUNNE \\ Haverford College, Haverford, Pennsylvania
}

\begin{abstract}
In two experiments, the effects of learning on both the accuracy and bias of duration judgments were examined. In Experiment 1, subjects learned one of two tasks (i.e., using a computer software package, building a model car), containing a varying number of action steps, over a one-, three-, or five-trial period. Retrospective judgments of a task's total duration revealed that accuracy was high at intermediate stages of learning but was low at early stages due to an overestimation bias and low at later stages due to an underestimation bias. The number of action steps within a task influenced behavior only at early learning stages where more action steps led to significantly longer duration estimates. Experiment 2 acted as a converging operation in which novice and experienced pianists were asked to estimate, in advance, how long they thought it would take them to play melodies that varied in their degree of familiarity (i.e. recently learned, well learned, extremely well learned). When these estimates were compared with the melodies' actual playing times, results revealed a similar pattern of accuracy and bias as found in Experiment 1. These findings are discussed in terms of a "structural remembering model" that emphasizes the role of event predictability in time estimation behavior.
\end{abstract}

In everyday behavior, there are many situations in which we rely on the memory of event durations. This is perhaps most evident in the planning and scheduling of a day's activities. For example, the hour at which we awake in the morning relative to the time we must be at work is, to a large extent, determined by our knowledge of how long it will take to shower and dress, drink our coffee, and navigate the morning traffic. Similarly, the particular activities initiated during a televised commercial break are often based on one's knowledge of how long an average commercial lasts. In addition to these judgments of events' expected durations, there are other occasions in which one estimates event durations in retrospect, such as, for example, how long it took to drive to a particular destination or to complete a given activity. In each of these instances, people appear to have a tacit knowledge of the inherent durations of different environmental events that is based on past experience with the events themselves. However, despite the frequency in which this type of knowledge is used, the learning and subsequent remembering of event durations have not been extensively investigated in the previous literature. Hence, it not clear what processing mechanisms are responsible for the acquisition of temporal knowledge or how duration accuracy and bias

This research was supported by a Faculty Research Grant from Haverford College and was presented at the annual Meeting of the Psychonomic Society in Chicago, November 1996. The authors wish to thank Douglas Nelson, John Wixted, and one anonymous reviewer for their critical comments on an earlier version of this manuscript, as well as Tara Colgan and Andrew Reznick for conducting a pilot study for Experiment 1 in partial fulfillment of their senior thesis requirement. Correspondence should be addressed to M. G. Boltz, Department of Psychology, Haverford College, Haverford, PA 19041 (e-mail: mboltz@ haverford.edu). may change with increased familiarity with a given event. The purpose of the present research was to investigate these issues through a set of laboratory experiments that were designed to assess time estimation behavior as people learn a novel motor skill.

\section{Models of Remembered Duration}

According to most theoretical models, the ability to accurately judge an event's duration depends on whether subjects' estimates are assessed in a prospective or retrospective fashion. This distinction in part refers to the particular instructions administered to subjects in an experimental context, but, more generally, it is assumed to reflect different types of cognitive processing activities. In prospective situations, subjects are informed in advance that a time judgment will later be required. Given that subjects are consciously monitoring time's passage, these estimates are therefore assumed to reflect experienced duration. In contrast, retrospective designs are assumed to reflect remembered duration because subjects do not know that a time judgment will be required until after an event has transpired.

Most theoretical frameworks assume that duration judgments will be quite accurate in prospective situations but not in retrospective ones. This idea, for example, is central to one of the most popular models in the current literature, an approach based on the resource allocation theory of selective attention (Kahneman, 1973; Navon \& Gopher, 1979). Zakay (1989) has developed one of the most comprehensive models by not only incorporating those ideas of other theorists but by integrating these with certain memory-based heuristics suggested to influence time estimation behavior (e.g., Block, 1985, 1989; Ornstein, 1969). According to this view, whenever subjects are engaged in an activity, attention is split between the process- 
ing of temporal and nontemporal information (i.e., task's duration vs. its set of actions). Each is assumed to be independently processed by the cognitive system but to nonetheless compete for a common pool of resources such that increased attention toward one dimension will decrease performance on the other.

Prospective situations should yield highly accurate time judgments because subjects are presumably focused on temporal information alone during the encoding phase and are free to engage in any conscious time-keeping strategy that may aid performance (e.g., counting, tapping, or monitoring the event's inherent rhythm). However, as task complexity increases, either due to greater difficulty or the performance of two concurrent activities, more resources will be directed toward the processing of nontemporal information. Duration estimates should therefore not only become more inaccurate and variable but should become shorter because less temporal information is being encoded into the cognitive system. Retrospective time judgments, on the other hand, are assumed to involve a very different set of processing activities. Here, there is no apparent reason to attend to event duration, and so most resources will be directed toward the processing of nontemporal information (e.g., action steps) in order to accomplish the task at hand. Relative to prospective designs, then, remembered duration judgments will be less accurate and more variable. In addition, increases in task complexity are predicted to yield an overestimation bias due to an "availability heuristic." This concept is one that has been borrowed from a second theoretical framework, known as the memory-based models (Block, 1985, 1989; Ornstein, 1969), which asserts that remembered duration is inferred from the amount of nontemporal information stored in memory. Given that people are unlikely to encode event duration under retrospective circumstances, they thereby rely on the only source of information that is available in memorynamely, the number of chunks (Ornstein, 1969) or changes (Block, 1985, 1989) within the array of nontemporal information. The more chunks or changes that are apparent, the longer time judgments will be.

This overall approach has received substantial empirical support. Relative to retrospective designs, prospective judgments have been found to be more accurate and less variable (Brown, 1985; Miller, Hicks, \& Willette, 1978). In addition, the results of two meta-analyses have revealed that increases in task complexity typically lead to shorter duration estimates in prospective designs but longer estimates in retrospective circumstances (Block \& Zakay, 1997; Zakay \& Meran, 1988, as cited in Zakay, 1989). However, there are other predictions of the model that are more problematical. In particular, it is assumed that event duration is only encoded into the cognitive system in prospective situations where people are consciously attending to time's passage. In contrast, people are presumably incapable of making retrospective judgments with any degree of accuracy. The problem with this assumption is that it is inconsistent with everyday experience. People do seem reasonably calibrated in the planning and scheduling of activities such that they are usually on time for the series of prearranged appointments that typically characterize one's day. In addition, one is often able to accurately judge the duration of a recently completed activity. These sorts of behavioral capabilities suggest that event duration may in fact be encoded into the cognitive system under retrospective circumstances.

\section{The Potential Impact of Learning}

One explanation for this discrepancy between the present literature and everyday experience may involve the influence of a variable that has been relatively unacknowledged in past research: that of learning. Many cognitive behaviors require repeated experience before a high level of performance is achieved, and the ability to accurately remember an event's duration may be subject to the same constraints. After all, when one estimates how long it will take to shower, dress, and make coffee in the morning, these estimates are based on daily experience acquired over the course of weeks, months, or years. With repeated exposure, memory for event durations should eventually become quite accurate and not only allow one to predict how long a given activity will take but also allow one to judge in retrospect how much time was spent performing the activity.

The variable of learning has in fact been extensively investigated in prospective situations. For example, several researchers have noted that the ability to accurately produce a given interval (e.g., $30 \mathrm{sec}, 1 \mathrm{~min}$, etc.) markedly improves over successive trials (Fraisse, 1971; Mohan, 1975; Montare, 1985, 1988). Similar findings have also been reported with the methods of reproduction (Fraisse, 1971; Fraisse \& Orsini, 1958) and verbal estimation (Montare, 1988). However, in contrast, few studies have examined the effects of learning on remembered duration judgments. To a large extent, this may be due to the way that the retrospective technique is typically implemented in an experimental context. Subjects are normally presented with a single exposure to a given event and then unexpectedly asked for a time judgment; because subjects are now aware of the experiment's purpose, any additional estimates would not be retrospective in nature. Given that past research has relied on this type of experimental methodology, this may explain why current theoretical approaches assume that people are incapable of producing retrospective judgments with any degree of competency. Without adequate learning experience, it is unsurprising that subjects' time estimates are so highly inaccurate and unreliable.

One way in which to assess the effects of learning on time estimation behavior is to modify the traditional use of the retrospective paradigm. In lieu of presenting subjects with a single event, it is possible to present several different events for a varying number of trials while subjects are engaged in a task unrelated to temporal experi- 
ence. Following this initial learning phase, subjects can then be given a surprise duration memory task in which they are asked to recognize, reproduce, or verbally estimate the duration of each event. This in turn not only allows one to obtain multiple time judgments from an individual subject but also allows one to compare performance across different groups of subjects who vary in their degree of familiarity with the set of events.

This type of procedure was developed and used in a series of experiments by Boltz (1992) in which the primary intent was to examine the effects of event structure on remembered duration. Subjects were first asked to perform a series of perceptual rating tasks on a set of musical patterns or environmental sounds that varied in their degree of internal predictability and coherence. These patterns contained a single pitch value that rhythmically recurred in time and were thereby coherent, were continuous in duration, or irregularly timed and thereby incoherent. After performing the set of rating tasks for a varying number of trials (four or eight), subjects were then unexpectedly asked to recognize or reproduce the total duration of the sounds heard earlier. Results showed that the durations of coherent and continuous events were relatively well remembered after four learning trials (recognition hit rates of $65 \%$ and $55 \%$, respectively) and performance significantly increased with greater learning experience (hit rates of $81 \%$ and $70 \%$, respectively). Incoherent events, on the other hand, yielded a very low level of accuracy (52\%), regardless of the number of learning trials.

Overall, these findings indicate that people are able to incidentally learn and remember the characteristic duration of events in retrospective situations, even though attending was presumably directed toward the events' nontemporal information during the encoding phase. This ability, however, occurs only when events are highly predictable in nature, a finding that has been corroborated in subsequent studies (Boltz, 1994, 1995, 1998a, 1998b). This effect has been interpreted within a framework known as the structural remembering approach, which, in contrast to other models, emphasizes the role of event structure in both attending and remembering (Boltz, 1998a).

\section{The Structural Remembering Approach}

The basic assumption of this model is that environmental events vary in their degree of structural coherence, which in turn determines both accuracy and bias of duration judgments. Highly coherent events are ones that display a very predictable arrangement of structure in which temporal and nontemporal information bear a lawful relationship to one another. In some cases, this can stem from an inherent rhythm such that the sequence of nontemporal items (e.g., words in an utterance, notes in a melody) unfolds with a lawful temporal periodicity. In other events, particularly those involving the performance of a given activity, predictability arises from not only knowing the relevant actions and their temporal sequence but the extent to which these are skillfully performed with no hesitations and a smooth coordination of body movements. In both instances, the overall predictability of the event arises from the joint relationship between temporal and nontemporal information. The sequence of nontemporal items (e.g., words, notes, action steps) unfolds in a temporally integrated fashion with a characteristic rhythm, rate, and total duration. Both structural dimensions jointly characterize the event and together provide a high degree of internal organization within the event's total time span.

From a cognitive perspective, highly coherent events facilitate attending and remembering in a number of different ways. First, given their overall degree of predictability, this enables one to easily track the event as it unfolds and to not only anticipate what information will occur but when in time it will appear. In addition, the joint structural relationship between temporal and nontemporal information allows both to be encoded into memory with the same amount of effort as required to input one dimension alone. Attending to one dimension will lead to an incidental learning of the other, regardless of which structural dimension was selectively attended to during the encoding phase. Contrary to the predictions of resource allocation theory, this also means that attending to both dimensions at once will not lead to a decrement in performance. Finally, structural coherence is predicted to facilitate memory performance. These types of events should be relatively easy to recapitulate, particularly when they display an apparent rhythm that can act as an effective retrieval scheme for remembering the event from beginning to end. This retrieval process should also reflect both the temporal and the nontemporal information, which jointly characterize the event. With respect to time estimation behavior, duration judgments are therefore predicted to be highly accurate in both prospective and retrospective situations. Even though subjects in the latter situation may be primarily attending to nontemporal information, the event's inherent temporal information (i.e., rhythm, rate, total duration, and temporal ordering of items) should nonetheless be incidentally learned to a comparable level of performance as intentional learning (i.e., prospective situations).

Less coherent events, on the other hand, are assumed to involve a very different set of mediational mechanisms. Here, the overall structural array is a very unpredictable one in which the sequence of nontemporal information appears ill-timed and temporally uncoordinated. This may arise from the temporal periodicity of successive items not being lawfully related to one another and thus yielding an overall arrhythmia. The music of a novice pianist, for example, is often characterized by this type of arrangement. Alternatively, the performance of a given activity may display multiple hesitations and pauses due to the uncertainty of not knowing which actions to perform next or how these should be executed. Given that there is no 
overall coordination of body movements that display a characteristic ordering, rhythm, rate, or duration, the event appears to contain a disconnected series of chunks that are temporally unrelated to one another. As a result, incoherent events will be very difficult to attentionally track and it will be difficult to anticipate their future course. More importantly, since the event's temporal and nontemporal information are structurally dissociated from one another, each must be selectively attended to independently of the other in order for encoding to occur. Although both dimensions can become available in memory, this will require more processing effort than needed for the encoding of coherent events. In addition, conditions of divided attending are predicted to lead to tradeoffs in performance such that increased attending toward one dimension will result in a decrement in the learning and remembering of the other. Hence, in terms of time estimation behavior, event duration should be well remembered only when it is selectively attended to during the encoding phase (i.e., prospective designs). As predicted by the resource allocation theory (Zakay, 1989), retrospective judgments should be highly inaccurate, and people may rely on the availability heuristic to infer event duration from the number of chunks or changes within the array of nontemporal information.

Some empirical support for these ideas has been observed. In a recent set of experiments by Boltz (1998a), subjects were presented with a series of coherent and incoherent melodies and instructed to attend either to the tunes' temporal (i.e., rhythm or total duration) or nontemporal (i.e., pitch relations) information alone during the initial learning phase, or both in tandem. When subjects were later asked to reproduce each melody's total duration, results indicated that the time spans of coherent melodies were remembered with a high degree of accuracy, regardless of which structural dimension(s) was attended to during the encoding phase. In contrast, the duration judgments of incoherent melodies were accurate only when subjects attended to duration during encoding (i.e., prospective situations). Performance was highly inaccurate and unreliable when subjects attended to the melodies' nontemporal (pitch) information and even more so when attention was divided between temporal and nontemporal information.

In sum, then, the structural remembering approach extends the resource allocation theory by illustrating that the ability to accurately remember event durations depends on the degree of predictability within the environment. Although less coherent events do require independent processing resources, as traditionally has been assumed, the same is not true of more predictable ones. Here, there is no need to independently attend to the events' temporal and nontemporal information because, on a structural level, they are lawfully and inextricably entwined. Both dimensions are therefore jointly encoded into memory such that attending to one dimension results in the incidental learning of the other. Overall, this means that, un- like the durations of incoherent events, those of coherent events can be accurately remembered in both prospective and retrospective circumstances.

\section{EXPERIMENT 1}

The purpose of the present research was to further investigate these ideas by more closely examining remembered duration judgments as a function of learning. In particular, it may be the case that the different mediational processes observed for events varying in their degree of internal coherence also occur at different stages of learning. After all, one result of learning is to render an event more familiar and more predictable over time. For example, consider the experience of learning to drive a car. Initially, this process seems to be a very bewildering one because it is not clear what exactly is required and what steps must be executed relative to others. However, after repeated experience, the individual action steps are performed in their appropriate temporal sequence and are lawfully related to one another by a smooth coordination of body movements that unfold with a characteristic rate, rhythm, and duration. In short, the sequence of nontemporal information (e.g., action steps) unfolds with an identifiable and lawful temporal structure. In this sense, then, early stages of learning are comparable to incoherent events, whereas later stages are analogous to highly predictable ones. If so, this suggests that retrospective time estimates should systematically vary with learning. Given the relatively high degree of unpredictability at early stages of learning, temporal and nontemporal information may be encoded in an independent fashion. Although duration should be well remembered in prospective situations, the same will not be true for retrospective judgments. Here, there is no apparent reason to attend to duration, and so estimates should not only be highly inaccurate but may be biased by the relative number of such steps or items (i.e., the availability heuristic). As events become more familiar, this bias should disappear, such that judgments are relatively accurate and less variable. At this point, the individual actions are able to be executed in a temporally coordinated fashion. If this in turn leads to a joint encoding of temporal and nontemporal information, then duration judgments should be quite accurate even in retrospective contexts.

These ideas were tested in Experiment 1. Subjects were asked to learn a novel motor activity consisting of several independent steps. Two different tasks were used for purposes of generality, and they involved learning to use a software package on the computer and building a model car from an Erector Set. In order to examine whether duration judgments are influenced by the relative number of segments within an event, two different versions of each task were developed such that one contained more steps than the other. In addition, the number of learning trials was manipulated (one, three, or five) between subjects to assess a relatively broad range of experience but 
one that would also not induce excessive boredom. Afterwards, all subjects were unexpectedly asked to verbally estimate the duration of the final trial.

\section{Method}

\section{Design and Subjects}

The design was a $2 \times 3 \times 2$ between-subjects factorial. The subjects were required to learn one of two tasks (using a computer software package or building a model car) over a varying number of trials (one, three, or five). There were two versions of each task, one containing a greater number of action steps than the other.

One hundred forty-four subjects from an introductory psychology course at Haverford College participated in the experiment for course credit. Twelve subjects were randomly assigned to one of the 12 between-subjects conditions.

\section{Apparatus}

For the model building task, an "Indy Car" Erector Set (Model 2203, manufactured by the Meccano, Inc., 1675 Broadway, NY, NY 10019) was used that consisted of 16 metal parts and 16 nuts and bolts that formed the main chassis of the car along with two metal axles, four plastic tires, and a small wrench and screwdriver.

The second task relied on the Statistica software package (manufactured by the StatSoft, Inc., 2325 East 13th St., Tulsa, OK 74104 ) that was used on a Macintosh Performa $6115 \mathrm{CD}$ computer.

\section{Procedure}

The subjects in all conditions were individually tested and participated in one of the following task procedures during the initial learning phase of the experiment.

Erector Set. The subjects were initially asked whether or not they had assembled an Erector Set during the past 12 years, and, if so, they were dismissed from the experiment. The remaining subjects were informed that they would be participating in a learning experiment and would later be asked a series of questions. Given that they would be using their hands, they were asked to remove all rings, watches, and bracelets so these would not interfere with their motor performance. At this point, they were shown a preassembled version of the car and told to study it for the next minute because they would be asked to build one like it over the course of the experiment. The subjects in the long version of the task were then given the complete set of individual parts and told to begin assembling the car, using the completed one in front of them as a prototype. In the short-task condition, the subjects received a similar set of instructions but were given four preassembled pieces that formed the main chassis of the car, along with the set of axles and wheels. After completing the model, the subjects in the three- and five-trial conditions were immediately given the same set of parts as before and asked to assemble the car again on two or four additional occasions. Throughout the course of the learning phase, the experimenter was present in order to unobtrusively time and record the actual duration of each trial with a stopwatch.

Computer software program. After ensuring that none of the subjects had previously used the Statistica program, the subjects were told that the purpose of the experiment was to investigate students' ability to learn a new software package for statistical analysis. As in the previous task, they were asked to remove all jewelry from their wrists and hands so these would not interfere with their motor performance. Given that these subjects were all introductory psychology students, they were first given a brief written tutorial on the underlying logic of the experimental method and the purpose of the $F$ test. This tutorial was very similar to a lecture that they had previously received in class, where they had reviewed the concepts of independent and dependent variables and the meaning of the $F$ statistic and $p$ levels. In addition, an exemplar experimental design was given. It described a hypothetical study in which males and females were compared for differences in pain sensitivity as measured by the response latency to a hotplate. The subjects were told that several data sets had been obtained and needed to be statistically analyzed with an $F$ test. At this point, the Statistica software package was introduced. The subjects were first given a verbal overview of what information they would have to provide to the program and were then taken through an exemplar data set on the computer itself. In the long version of the task, this involved the following: creating a data file in which the codes for the male and female levels of the independent variable were entered into the first column of the spreadsheet, and the values for the dependent measure of latency were entered into the second; saving this file and transferring to the analysis-of-variance module of the program where the number of subjects in the experiment and the names of both independent and dependent variables were specified; retrieving the means and standard deviations for each group and then the results of the $F$ test; and, creating a graph that depicted the mean latency difference between the two gender groups, which also involved rescaling the $y$-axis, adding labels to axes, and a figure caption. The subjects in the shorter version of the task performed these exact same steps except for the retrieval of the means and standard deviations and the creation of a graph. After going through this exemplar data set with the experimenter, all subjects were then given a second data set and told to analyze this on their own with the procedure that had just been illustrated-a process that was repeated again in the three- and fivetrial conditions. Again, the experimenter was always present in the room to discretely time and record the actual duration of each individual trial.

Memory phase. Following the initial learning phase, all subjects were asked to complete a questionnaire that assessed several different indices of behavior. They were first asked to estimate, to the nearest half minute, how long it took to complete the last trial of their respective task and to then estimate the duration of each preceding trial. After this, they were asked to describe and clearly identify each individual step of the task in which they had just participated. The fourth and fifth questions asked them to rate their interest in the task on a 7-point scale $(1=$ very interesting $)$ and how difficult they found the task to be $(1=$ very easy). The subjects who had learned the computer software package were given two additional questions: (1) how many hours per week they typically used a computer, and (2) how they would rate their overall enjoyment level in using computers on a 7 -point scale $(1=$ very enjoyable $)$.

\section{Results}

\section{Actual Duration of Tasks}

Before evaluating the subjects' duration estimates, a preliminary set of analyses was conducted to determine whether learning actually occurred across the different trials of the experiment. If so, then one would expect the actual duration required to perform a task to gradually decrease between the one-, three-, and five-trial conditions. An overall between-subjects analysis of variance (ANOVA) revealed this was the case. A main effect for trials $\left[F(2,132)=27.96, M S_{\mathrm{e}}=0.84, p<.001\right]$ indicates that the subjects in the one-trial condition took significantly more time to complete their respective task than did those in the three-trial condition, who in turn took significantly more time than did the subjects in the fivetrial condition. Although this learning effect occurred 
for both tasks, a significant interaction between task and trials $\left[F(2,132)=8.18, M S_{\mathrm{e}}=0.84, p<.001\right]$ shows that the difference between trials was greater for the subjects building a model car $(M \mathrm{~s}=18.04,7.54,6.61$, for one-, three-, and five-trial groups, respectively) than those analyzing data on the computer $(M \mathrm{~s}=5.86,4.38,3.98$, respectively). Overall, the Erector Set required a longer duration than did the computer software package $[F(1,132)=$ $\left.12.32, M S_{\mathrm{e}}=0.84, p<.001\right]$, and, unsurprisingly, the tasks containing a greater number of steps took longer than did those with fewer steps $\left[F(1,132)=44.80, M S_{\mathrm{e}}=\right.$ $0.84, p<.001]$.

A second set of analyses examined the change in a task's duration for the subjects within the three- and fivetrial conditions. For each group, there was a significant main effect for trials $\left[F(2,33)=7.49, M S_{\mathrm{e}}=0.66, p<\right.$ .01 , for three-trial group, and $F(4,55)=9.2, M S_{\mathrm{e}}=0.47$, $p<.01$, for the five-trial group] such that the subjects became increasingly faster in performing their respective tasks over successive trials.

\section{Duration Estimates}

Accuracy and bias between subject groups. In order to determine both accuracy and bias of duration judgments, a ratio score was calculated for each subject that evaluated the actual duration of the last learning trial relative to its estimated duration $(E D / A D)$. Ratios equal to 1 therefore reflect perfect accuracy, whereas those greater than 1 indicate relative overestimations, and those less than 1 indicate relative underestimations. One can also determine the mean percent error in the subjects' estimates by subtracting a value of 1 from each observed ratio and then multiplying by 100 . For example, a ratio of 1.30 indicates that the subjects, on average, overestimated a task's duration by $30 \%$. These mean ratio scores are shown in Figure 1 as a function of the number of learning trials, relative number of task steps, and task type. This first analysis focused exclusively on time estimates of the final learning trial in order to directly compare performance between the different subject groups.

An overall ANOVA revealed three major findings. First, a main effect of learning trials $[F(2,132)=23.25$, $\left.M S_{\mathrm{e}}=0.13, p<.001\right]$ indicates that error and response bias systematically changed with greater experience. At early stages of learning, judgments were significantly overestimated on the order of $39.5 \%$, and this effect applied to $85 \%$ of all subjects. Bias then disappeared after three learning trials where ratios did not vary from a value of 1 . However, bias again reappeared with additional learning, but this time in the form of underestimations where judged duration was on average $28 \%$ shorter than a trial's actual duration. This too was a relatively robust finding that applied to $66 \%$ of all subjects.

This pattern of error and bias generalized to both conditions of task segmentation, but an interaction of task segments with number of learning trials $[F(2,132)=$ $\left.11.47, M S_{\mathrm{e}}=0.13, p<.001\right]$ indicates that bias was more pronounced in some conditions than in others. As shown in Figure 1, an increase in the number of task steps at early stages of learning led to a significantly greater degree of overestimation. At later stages, however, the magnitude of error and bias was comparable across the two segmentation conditions. These differences were confirmed by a set of Tukey post hoc comparisons $(p<.05)$.

Although these effects apply to both task types (shown in Figures 1A and 1B), a significant two-way interaction between number of learning trials and task type $[F(2,132)=$ $\left.6.57, M S_{\mathrm{e}}=0.13, p<.01\right]$ indicates that the degree of overestimation bias was significantly greater in the one-trial condition for the computer software task.

Accuracy and bias within subjects. A second analysis was performed to evaluate whether the observed pattern of error and bias between the different subject groups also applied to within-subjects conditions. That is, for the subjects in the three- and five-trial conditions, ratio scores were obtained for each successive trial. Overall, the same findings as before emerged. These are shown in Figure 2 for the three-trial group and in Figure 3 for the five-trial group.

Within each group, an overestimation bias appeared at early stages of learning, gradually disappeared, and then transformed into an underestimation bias at later stages. This main effect of trials was significant $[F(2,33)=9.17$, $M S_{\mathrm{e}}=0.01, p<.01$, for the three-trial group, and $F(4,55)=$ $8.77, M S_{\mathrm{e}}=0.08, p<.01$, for five-trial group], and, again, there was a two-way interaction of trials and task segments $\left[F(2,132)=6.83, M S_{\mathrm{e}}=0.01, p<.01\right.$, and $F(4,132)=7.14$, $M S_{\mathrm{e}}=0.08, p<.01$, for the three- and five-trial groups, respectively] such that the overestimation bias after one trial of learning was significantly greater when there were more task steps. Lastly, this latter effect was more apparent in the computer software task, which in turn yielded a significant three-way interaction between number of learning trials, relative number of task steps, and task type $\left[F(2,132)=5.41, M S_{\mathrm{e}}=0.01, p<.01\right.$, and $F(4,132)=8.19, M S_{\mathrm{e}}=0.08, p<.01$, for the three- and five-trial groups, respectively].

In sum, then, the effects of learning and task segmentation on duration judgments were very reliable ones that applied to both between- and within-subject conditions.

Covariate analyses. The rating data were also evaluated. The mean difficulty ratings produced no significant effects and yielded an overall mean of 3.22 across all conditions of the experiment. Mean interest in the two tasks, however, varied as a function of trials to yield a significant main effect $\left[F(2,132)=3.77, M S_{\mathrm{e}}=1.67, p<\right.$ $.03]$. Although the subjects in the one-trial condition found the tasks to be somewhat interesting $(M=3.21)$, this interest level declined after three trials $(M=3.45)$ and even more so after five trials $(M=4.23)$. An analysis of covariance (ANCOVA), however, indicated that this effect was independent of the judged duration data.

For the subjects participating in the computer software task, the average amount of computer use per week 


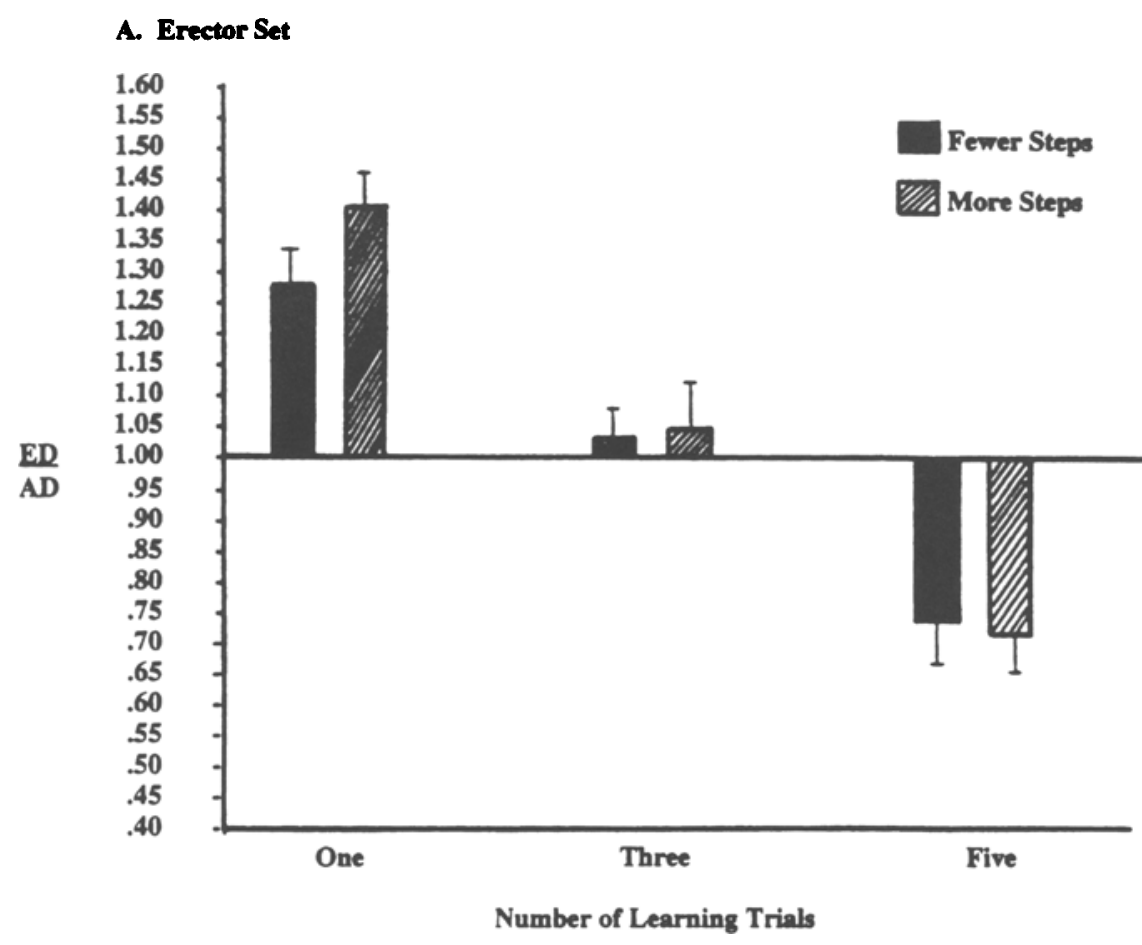

\section{B. Computer Software Pecloge}

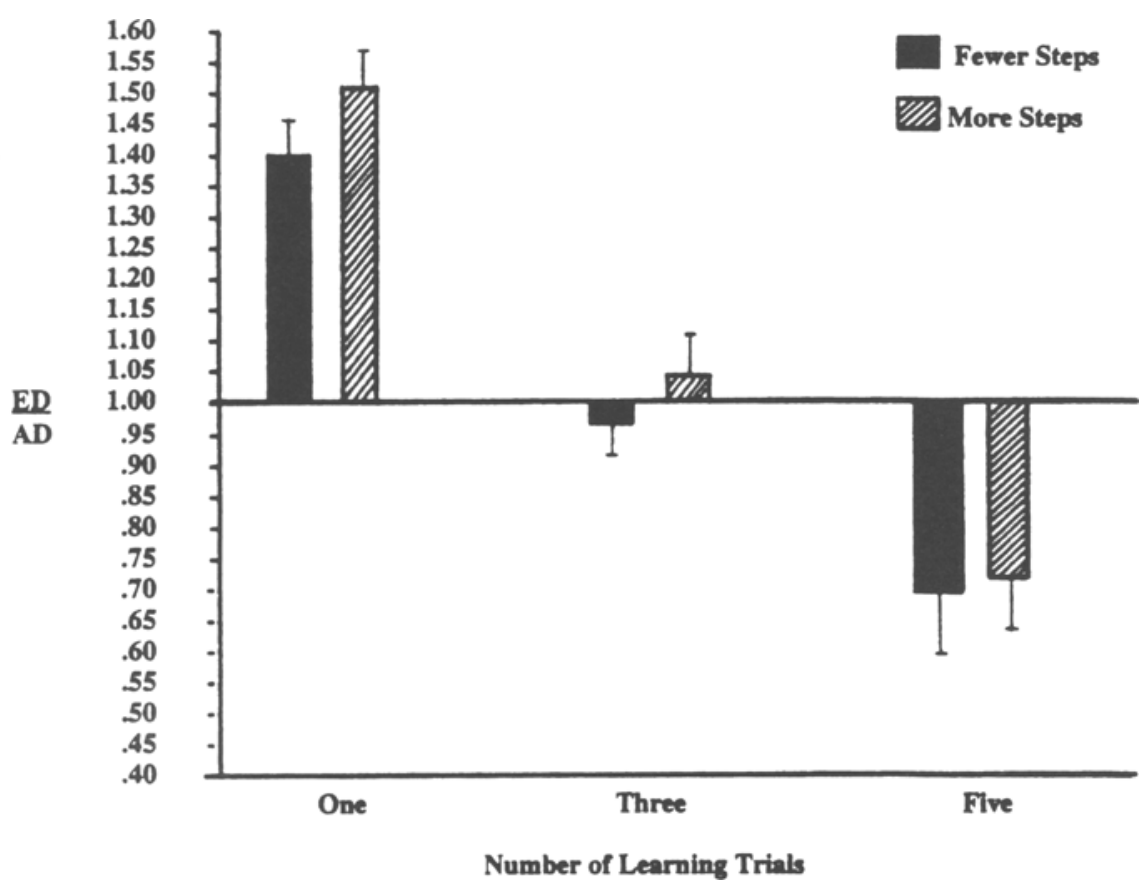

Figure 1. Mean ratio values (ED/AD) in Experiment 1 as a function of the number of learning trials, relative number of task steps, and task type.

was analyzed, along with the judged enjoyment level of using computers. Both variables produced null effects and were comparable across all experimental conditions ( $M=8.8 \mathrm{~h}$, and $M=3.00$, respectively). In addition, they did not exert any influence on duration estimates.

\section{Estimated Number of Task Steps}

A final analysis was conducted to determine whether the perceived number of task steps varied as a function of the experimental manipulations. These mean estimates are shown in Figure 4. 


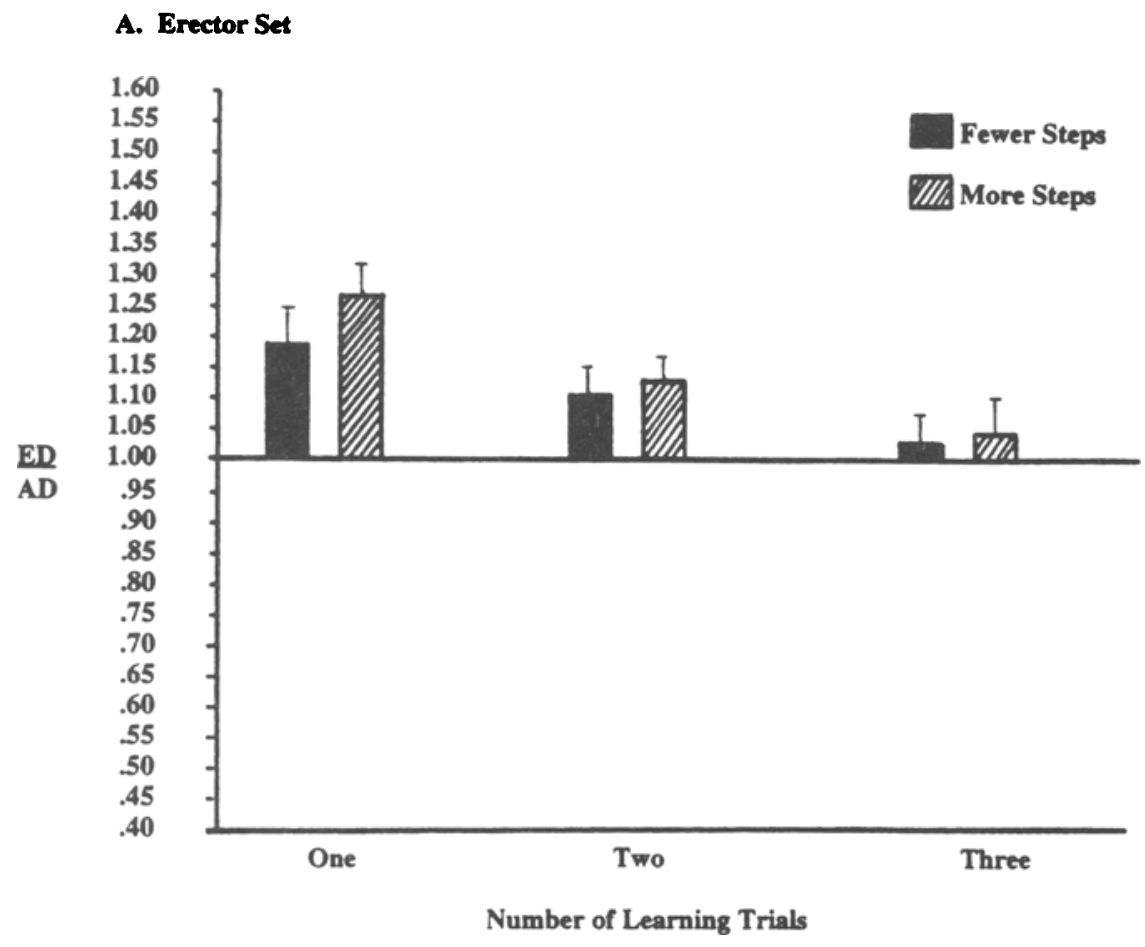

\section{B. Computer Software Package}

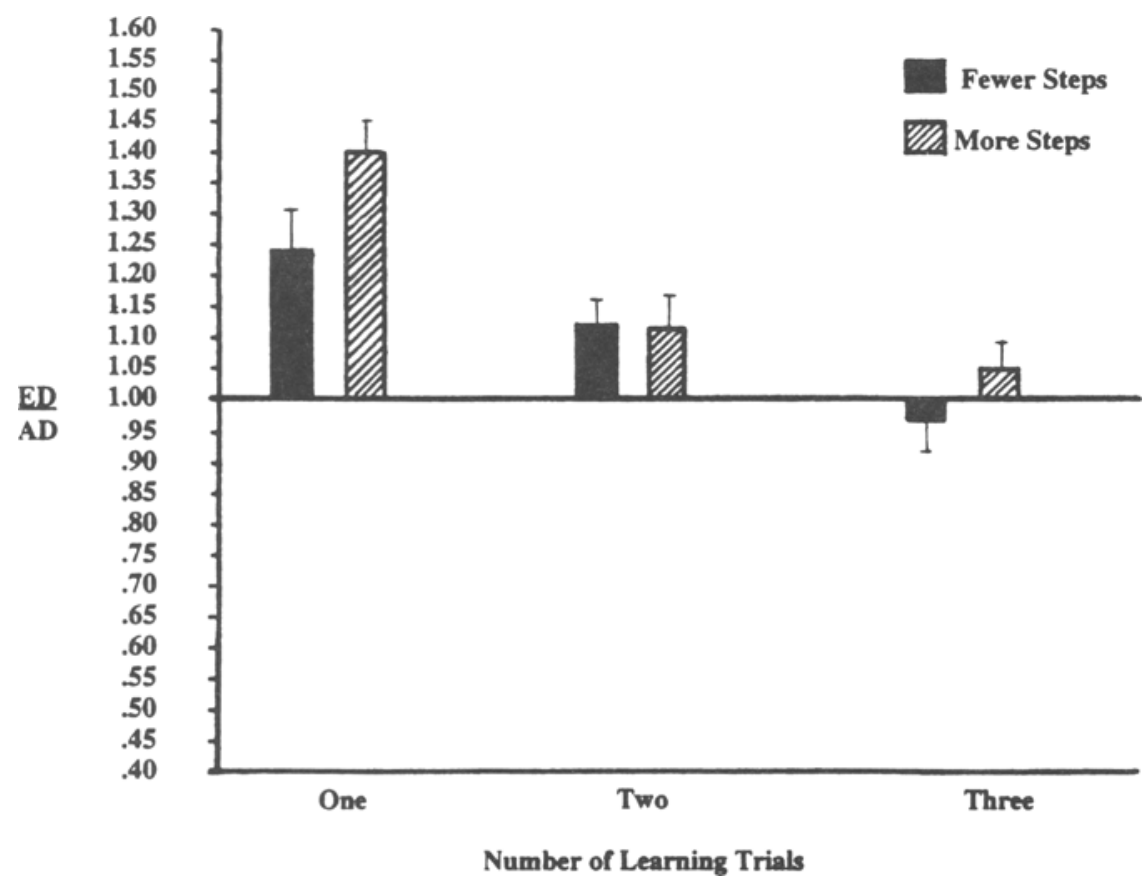

Figure 2. Mean ratio values (ED/AD) for the subjects in the three-trial condition of Experiment 1 as a function of the number of learning trials, relative number of task steps, and task type.

The most interesting finding was a main effect for learning $\left[F(2,132)=7.42, M S_{\mathrm{e}}=4.69, p<.01\right]$. A set of Tukey post hoc comparisons indicates that the number of estimated steps significantly decreased as the amount of experience on a task increased $(p<.05)$. This effect applies to both tasks; however, as seen Figure 4, the computer software task was judged to contain an overall greater number of steps than the Erector Set $[F(1,132)=$ 


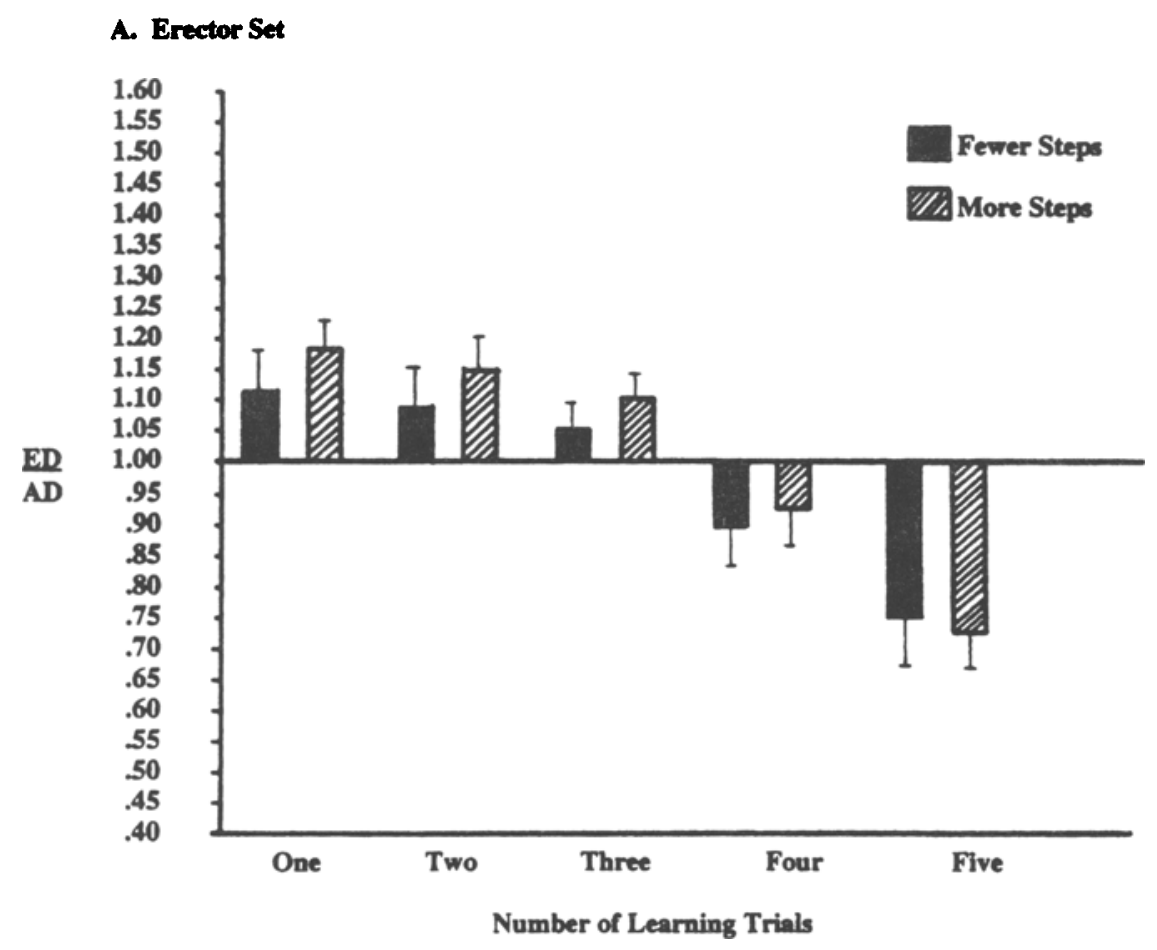

B. Computer Software Package

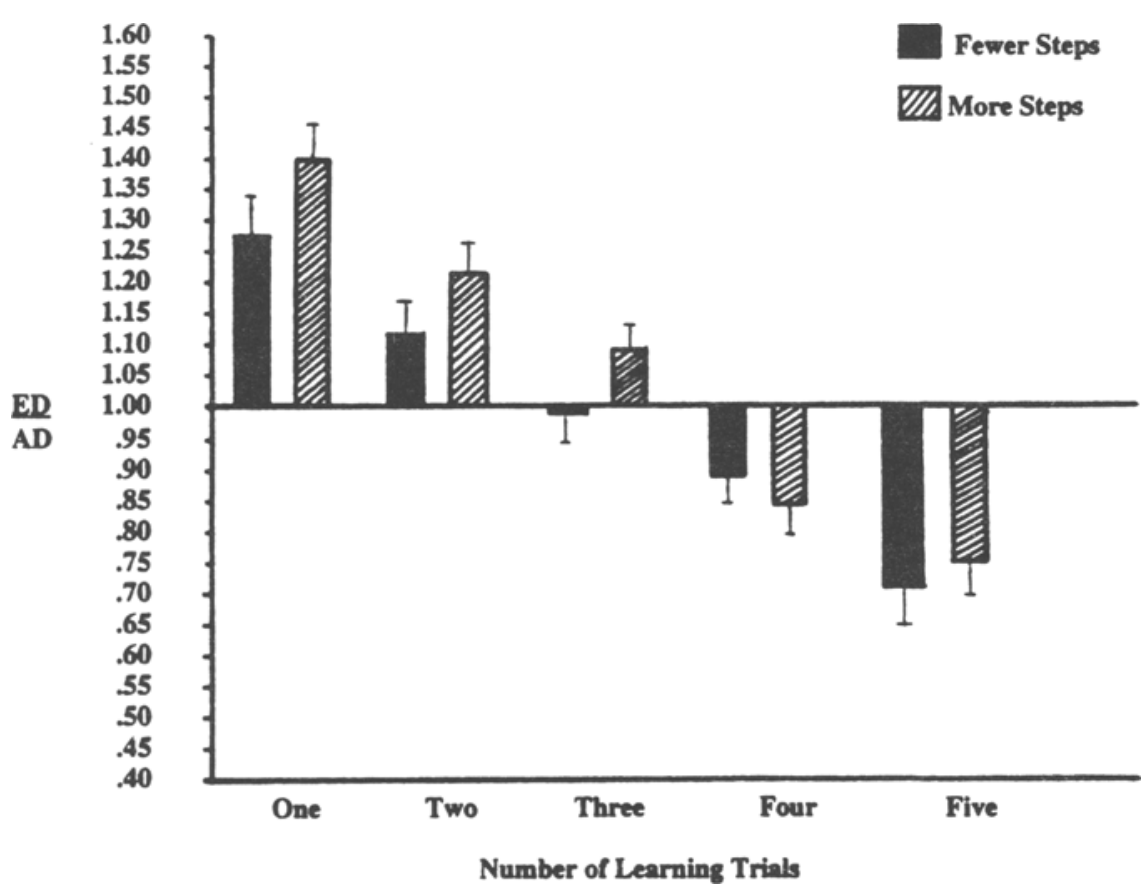

Figure 3. Mean ratio values (ED/AD) for the subjects in the five-trial condition of Experiment 1 as a function of the number of learning trials, relative number of task steps, and task type.

$\left.8.63, M S_{\mathrm{e}}=4.69, p<.01\right]$. Unsurprisingly, there was a main effect for task segmentation $[F(1,132)=16.92$, $\left.M S_{\mathrm{e}}=4.69, p<.001\right]$ in which the longer version of each task was considered to contain more individual steps than the shorter one. Lastly, a two-way interaction between task type and task segmentation $[F(1,132)=6.39$, $\left.M S_{\mathrm{e}}=4.69, p<.01\right]$ indicates that the difference between the short and long versions of each task was significantly 


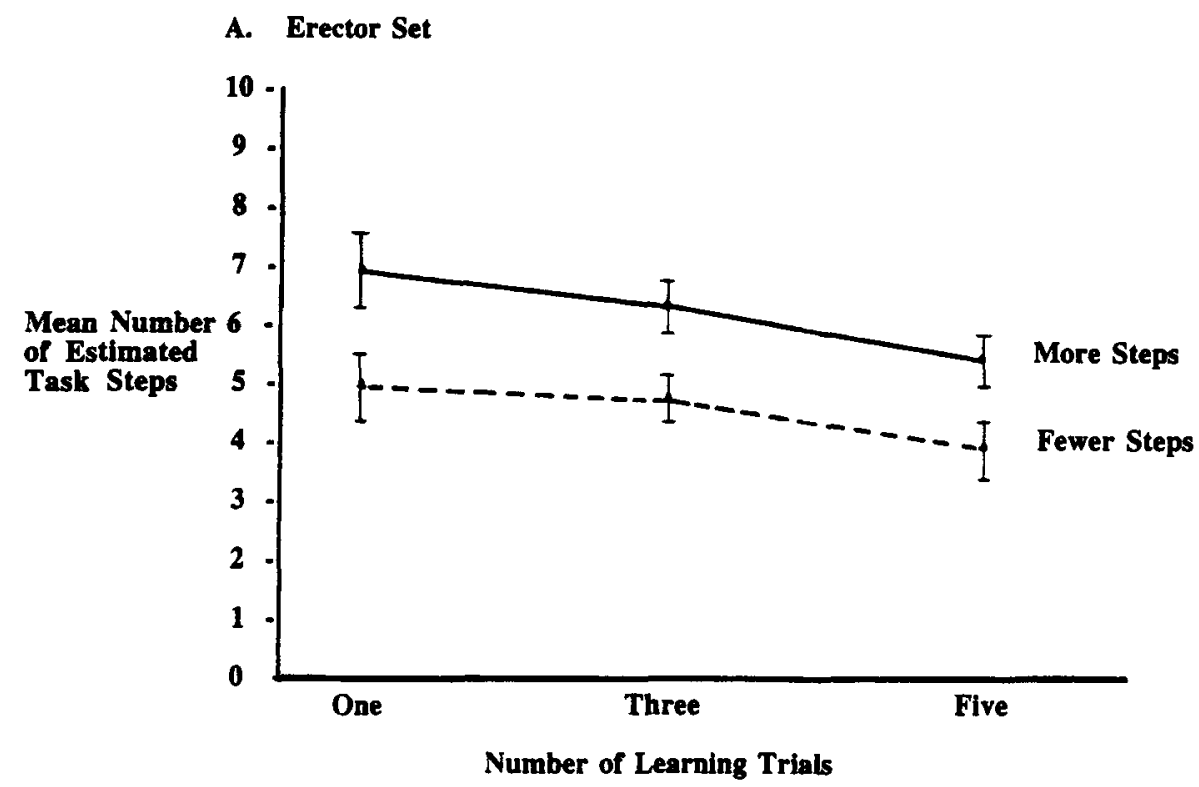

B. Computer Software Package

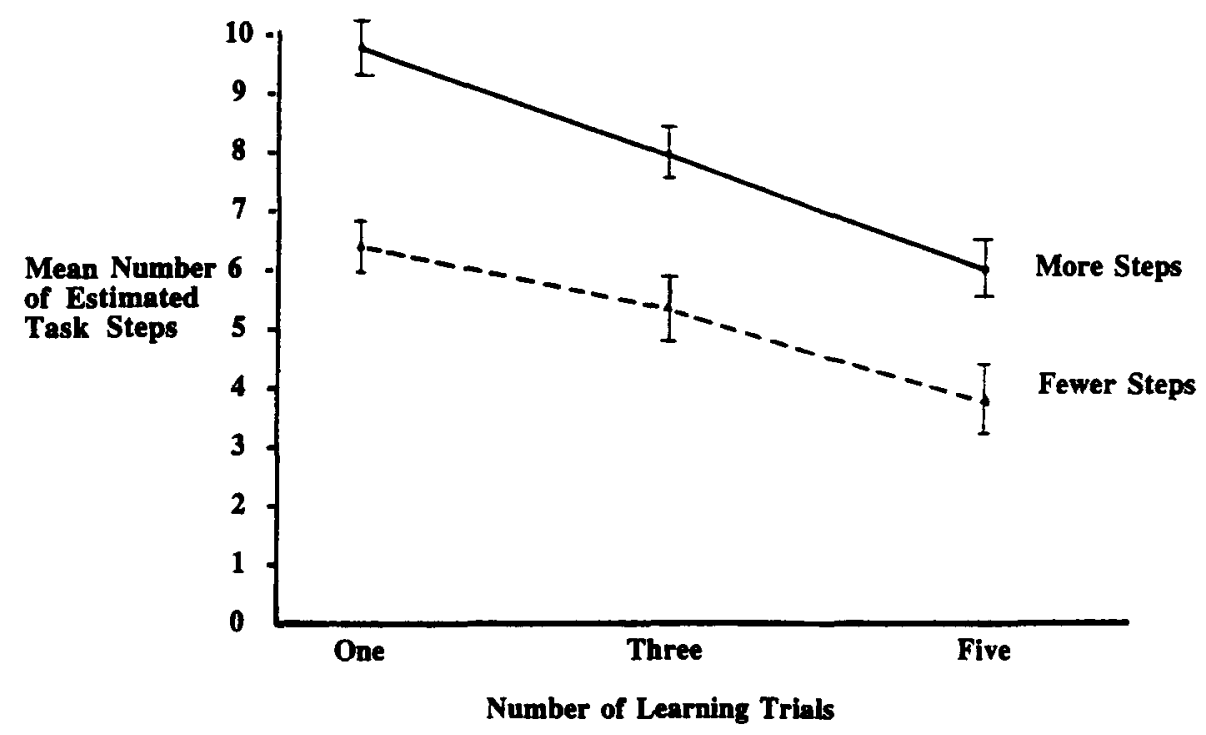

Figure 4. Mean number of estimated task steps in Experiment 1 as a function of the number of learning trials, relative number of task steps, and task type.

greater for the computer software task than for the Erector Set.

\section{Discussion}

As a set, the results of Experiment 1 render several insights into the nature of remembered duration. First, whereas most theoretical approaches assume that retrospective duration estimates are highly inaccurate and variable, the present findings suggest that they are quite orderly. The estimates may be too long, perfectly accurate, or too short depending on the degree of learning a factor that has generally been ignored in past research.
From a cognitive perspective, learning serves to render a higher degree of familiarity and predictability to an event's structure. When one first encounters a novel activity, its sequence of requisite steps will appear relatively independent of one another because it is not yet clear how these should be performed and in what temporal order. In order to encode both temporal and nontemporal information into memory, each dimension must therefore be selectively attended to independently of the other. In retrospective situations where most attentional resources are directed toward nontemporal information, event duration is unlikely to be encoded into 
memory. Hence, when people are later asked to produce a temporal judgment, estimates will not only be highly inaccurate but may reflect the use of an availability heuristic in which duration is inferred from the relative number of chunks or changes within the task itself. If so, then the tasks containing more segments should seem relatively longer than those with fewer segments. Evidence for this type of strategy was observed in Experiment 1. Accuracy was very poor after one trial of learning, and judgments became increasingly overestimated as the number of steps increased in the longer version of each task. This was particularly true in the computer software task, which was judged to contain more individual steps than the Erector Set. Overall, then, these findings are consistent with the findings of other studies showing that retrospective time judgments are biased by the amount of segmentation within an event (Poynter, 1983; Zakay, Tsal, Moses, \& Shahar, 1994).

What is novel here is the fact that this bias disappears to yield a high level of accuracy with additional learning experience. At this point, people know what exactly is required of them and are able to perform the requisite set of subactions in a temporally integrated fashion. This characteristic is one that differentiates a novice from an expert in any given field (e.g., de Groot, 1966; Egan \& Schwartz, 1979), and it was displayed here in the estimated chunk data: In both tasks, the perceived number of action steps decreased as the number of learning trials increased. More importantly, the high degree of predictability within the event's structure leads to a joint encoding of both temporal and nontemporal information such that event duration can be incidentally learned and remembered even when it is not selectively attended to.

In sum, then, these overall effects of learning on duration judgments are directly analogous to those resulting from manipulations of event structure. Early stages of learning display a high degree of uncertainty and yield a similar pattern of behavior as found in the judged duration of ill-timed melodies or environmental sounds. Similarly, the level of accuracy that results from greater learning experience is comparable to that found with highly rhythmic events (Boltz, 1992, 1995, 1998a). Despite these different origins, structural coherence provides the common function of enabling individuals to jointly encode temporal and nontemporal information into memory such that event duration can later be accurately remembered even in retrospective situations.

The final result of interest in Experiment 1 is the level of performance observed at later stages of learning. Although the structural remembering approach predicted that a high level of accuracy would maintain with extensive learning experience, a significant underestimation bias emerged. This effect occurred not only in each task but both between and within subject groups. In addition, it also appeared despite the overall decrease in subjects' interest level, which typically results in overestimations of an event's time span (Loehlin, 1959). At least two different phenomena may be at play here. One is that, with overlearning, subjects may be omitting certain lower level details when reconstructing the event's duration from memory. That is, some action steps may be so highly automaticized that they are simply "forgotten" during remembering. Some support for this idea comes from the estimated chunk data in which the number of perceived steps within a task was found to significantly decrease from the one to five trial learning conditions. The other possibility involves overconfidence. In the human performance literature, it has frequently been observed that subjects' estimates of their own abilities exceed their actual performance levels (Adams \& Adams, 1961; Oskamp, 1962). A similar process may be occurring here. After performing the task on five successive occasions, the increased sense of ease that results may lead subjects to misremember doing the task faster and more efficiently than what was actually the case. This phenomenon in fact may be operating in tandem with the omission of certain lower level details in remembering.

\section{EXPERIMEN'T 2}

The primary goal of Experiment 2 was to determine whether the effects of learning on duration judgments generalize to a different methodological context. In particular, an alternative way that temporal knowledge is frequently used in everyday behavior was considered. To date, much of the past literature has assessed duration judgments immediately after an event has transpired. This strategy, in fact, was used in Experiment 1, where the subjects judged a task's duration at the end of an experimental session. These types of studies are useful because they enable one to determine the accuracy with which people are able to monitor time's passage in prospective situations or remember the duration of a preceding event in retrospect. However, in addition to these types of judgments, event durations are also frequently used for planning and scheduling purposes. For example, if one has several errands to run before going to work (e.g., stopping by the bank, post office, drugstore, etc.), then one must tacitly estimate in advance how long each activity will take in order to determine when to leave the house. In these situations, then, people are generating expected duration judgments for a set of events that has not yet transpired. These are at least as common as estimates of experienced or remembered duration, because our daily lives often consist of a series of activities that are scheduled within particular time slots. Given this, people should be motivated to achieve a reasonable degree of accuracy, because different types of errors will result in negative consequences: Underestimations will lead one to be late for a subsequent activity, and overestimations will lead to an overabundance of time with perhaps no meaningful activity to fill this interval

Although expected duration judgments have not been well investigated in the previous literature, there is at least one study that has examined this ability. Burt and Kemp (1994) asked a group of college students to esti- 
mate how long they thought it would take to perform different tasks (e.g., consulting the card catalog in the library, walking from one building to another, going to the bookstore to buy a stamp) and then compared these estimates to the tasks' actual durations. Overall, the results revealed a high degree of variability: Although the expected duration judgments of some tasks were quite accurate, others were not.

One factor that might have contributed to these findings but was unacknowledged is a subject's degree of learning experience. The ability to predict an expected duration appears in many ways akin to remembering duration in retrospect. However, in lieu of recapitulating an event after it has transpired, one must retrieve the sequence of requisite actions before they have been performed. In the case of familiar tasks that have been repeatedly executed, this retrieval should contain not only information on the individual action steps themselves but also the rate, ordering, and duration with which these are typically performed. In contrast, the set of actions within less familiar tasks will appear more disconnected from one another, and there will be uncertainty about their overall ordering and execution. Hence, temporal information is less apt to be available unless it was selectively attended to during previous task performances. The overall prediction, then, is that the accuracy of duration estimates should increase with greater learning. However, if the pattern of bias observed in Experiment 1 generalizes to expected duration judgments, then the direction of error may again vary with increased experience. That is, judgments at early stages of learning may be influenced by the relative number of action steps (i.e., availability heuristic) and thus display relative overestimations. Although bias should eventually disappear, it may reemerge at later stages of learning in the form of underestimations.

In addition to providing estimates of expected duration, this methodological context offers two additional indices of behavior that are useful to examine. First, subjects can be asked to estimate the duration of a task after it has been performed. Given that expected duration judgments had been requested earlier, these estimates are most likely prospective in nature, meaning that subjects may elect to engage in conscious time-keeping strategies to facilitate performance. Nonetheless, learning should contribute to the overall level of accuracy. The more interesting measure, however, derives from comparing these latter estimates with those of expected duration to assess whether there is any change in both accuracy and bias of behavior. This too may vary with learning experience. In contrast to novices, more skilled performers should be better able to assess the extent to which their initial estimate deviates from the task's actual duration and thus improve on the accuracy of their subsequent estimate.

These various issues were addressed in Experiment 2. In order to avoid any confounds or alternative interpretations of the data that may arise from the use of different tasks across different conditions of the experiment, the performance of only one type of task was examined: that of piano playing. In particular, a group of musicians was asked to first estimate how long they thought it would take them to play a given composition, to then play the comtake position itself, and, afterwards, to estimate the duration of this actual performance. The variable of learning was manipulated in two ways. First, all subjects were asked to perform a total of six different compositions, two that had been only recently learned and thus infrequently played, two that were extremely well learned and thus frequently performed, and two that were in between these two extremes. In addition, the subjects' degree of musical expertise was varied. Half of the subjects had learned to play the piano within the past 12 months, and the other half were relatively experienced musicians who had been playing for several years. These two manipulations together were expected to indicate whether the effects of learning on time estimation behavior simply depend on the overall frequency in which a particular composition has been performed in the past or whether results are also dependent on one's skill level.

\begin{abstract}
Method

\section{Design and Subjects}

The design was a $2 \times 3 \times 3$ mixed factorial. Two groups of subjects who varied in their piano-playing ability (novice, experienced) were asked to estimate the durations of three types of compositions that varied in their overall familiarity (recently learned, well learned, extremely well learned). The second between-subjects variable was the particular order in which the three types of compositions were judged (I, II, III).

Thirty-six students from Haverford College participated in the experiment, and each was paid $\$ 8$. Half were relative novices who were enrolled in an int roductory piano course during the current or preceding semester and were practicing on a regular basis. On average, they had 6 months of experience. The remaining subjects were more experienced pianists who had taken an advanced piano course, who were junior or senior music majors, or who were members of an instrumental group. Each also practiced on a regular basis and had at least 5 years of piano-playing experience, with an overall average of 11 years.
\end{abstract}

\section{Apparatus}

During an experimental session, each subject played their set of compositions on a Steinway baby grand piano. These performances were recorded onto cassette tape with a Realistic CTR-22 tape player.

\section{Prosedure}

The subjects were randomly assigned to one of the three counterbalancing conditions. They were first asked to complete a brief questionnaire that assessed several different indices of their past piano-playing experience (i.e., how long they have been playing, number of hours per week spent practicing, participation in any instrumental group). Following this, they were told that the purpose of the experiment was to investigate certain types of abilities people have after they have learned to play the piano. They were also told that they would be performing a series of tasks, but the nature of these was not revealed until immediately before a given task. At this point, the subjects were asked to provide the set of compositions that they had been asked to bring with them, all notated on sheet music. These pieces were ones they were free to select on their own, as long as each satisfied the following criteria: from the 
genre of classical music and containing both treble and bass lines, an underlying meter (tempo) in which the referent beat was either a quarter note $(\delta)$ or an eighth note $(\delta)$, and a song duration that ranged from $1 / 2$ to 3 pages in length. Most importantly, two songs were to be representative of the following three categories: songs that were recently learned $(R L)$ and thus infrequently played in the past, songs that were extremely well learned (EL) and their most frequently played pieces, and songs that were well learned (WL) but fell in the middle of these two extremes.

For each individual melody, the subjects then performed a series of judgment tasks. First, they were asked to estimate the total number of hours this particular piece had been practiced and, following this, to rate the melody on a 10-point scale for its degree of familiarity $(10=$ extremely well learned $)$, overall playing difficulty $(1=$ very easy to play $)$; and likability $(1=$ like very much $)$. These judgments were always performed as a set for a given melody in the sequence described, but the ordering of the melodies themselves was a randomized one.

In the second phase of the experiment, the time estimation data were obtained. For each melody, the subjects were initially asked to estimate, to the nearest half minute, "how long do you think it will take you to play this piece in its entirety, and at its natural tempo?"Next, the subjects actually played this piece on the piano, with the sheet music in front of them. They were told not to worry about making any mistakes and, if these occurred, to simply continue playing. The subjects were also informed that their performances would be recorded onto cassette tape so that these could later be judged for the appropriateness of their produced tempi. During the subjects' performance, the experimenter was always present to time the composition from beginning to end with a stopwatch that was hidden from view. Immediately afterwards, the subjects were asked to estimate, to the nearest half minute, how long they thought it took them to actually play the piece. This overall procedure was then repeated for the remaining melodies. The two songs within a given learning category were always blocked together, but the categories themselves were arranged into one of three counterbalance orders: RL, WL, EL; WL, EL, RL; EL, RL, WL. In addition, the subjects were not given any feedback on the accuracy of their duration judgments until after the entire experimental session had ended.

In the final task, the subjects were presented with a copy of the sheet music from which they had performed earlier, and, for each composition, they were asked

to indicate the natural segments of the piece, as you think of them in your own mind. That is, as you are playing the piece, you may think of it as consisting of different sections. Please mark an $X$ at the ending of each of these sections.

The subjects were tested individually, and the duration of an entire experimental session was approximately $1 \mathrm{~h} 15 \mathrm{~min}$.

\section{Results}

\section{Learning Measures}

In order to determine whether the set of compositions selected by the subjects actually varied in their degree of familiarity, two types of judgments from the first phase of the experiment were evaluated. The first was the total number of hours each melody had previously been practiced. The results of an overall ANOVA revealed that, unsurprisingly, the experienced musicians had spent more time practicing their set of melodies than had the novices $\left[F(1,30)=36.96, M S_{\mathrm{e}}=150.59, p<.0001\right]$. Nonetheless, a main effect for tune familiarity $[F(2,60)=142.54$, $\left.M S_{\mathrm{e}}=121.52, p<.0001\right]$ indicated that, across both groups of subjects, recently learned tunes were practiced for a fewer number of hours $(M=4.05)$ than well learned ones $(M=14.58)$, which in turn were practiced less than extremely well learned melodies $(M=34.58)$.

The set of familiarity ratings revealed a similar pattern of results. Here, a main effect for tune familiarity $\left[F\left(2,60=270.2, M S_{\mathrm{e}}=2.20, p<.0001\right]\right.$ confirmed that melodies were judged increasingly more familiar as the recency in which the tune had been learned decreased ( $M \mathrm{~s}=2.25,5.34,7.99$, respectively).

Overall, then, these data suggest that the three types of melodies selected by the subjects did in fact vary in their degree of past learning experience, which thereby validates the main manipulation of the experiment.

\section{Duration Estimates}

Several different indices of time estimation behavior were examined. The main measures of interest were accuracy and bias of expected duration judgments. However, the subjects' estimates of their actual playing times were also analyzed, as was the degree of change in these latter estimates relative to the initial judgments of expected duration. Each is discussed in turn. In all cases, the subjects' data are collapsed over counterbalance order and the two instances within a given tune category.

Accuracy and bias of expected duration judgments. The directional error of the subjects' expected duration judgments was again assessed through a set of ratio scores that evaluated a melody's expected duration relative to its actual duration (i.e., EXD/AD). These mean values are depicted in Figure 5 as a function of tune familiarity and subjects' degree of musical expertise.

An overall ANOVA reveals one significant finding: a main effect for tune familiarity $\left[F(2,60)=42.82, M S_{\mathrm{e}}=\right.$ $0.187, p<.0001]$. Across both subject groups, notice that the most recently learned tunes were overestimated on the order of $48 \%$. Error then significantly declined with greater learning experience such that melodies, on average, were now judged to be only $11 \%$ longer than their actual duration. However, a different type of bias appeared with melodies that had been extremely well learned: Here, significant underestimations occurred on the order of $19 \%$.

Accuracy and bias of postperformance estimates. A similar analysis was conducted to evaluate the subjects' postperformance duration relative to a melody's actual playing time (i.e., PPD/AD). In general, the results closely converged with the subjects' expected duration judgments.

Mean ratio scores are shown in Figure 6 as a function of tune familiarity and musical expertise. Results from an overall ANOVA indicate that bias systematically changed with tune familiarity $\left[F(2,60)=9.76, M S_{\mathrm{e}}=\right.$ $0.092, p<.002]$. Recently learned melodies yielded significant overestimations in which melodies, on average, were judged $18 \%$ longer than their actual durations. This bias then disappeared with well-learned tunes for which ratios did not vary from a value of 1 . However, bias again 


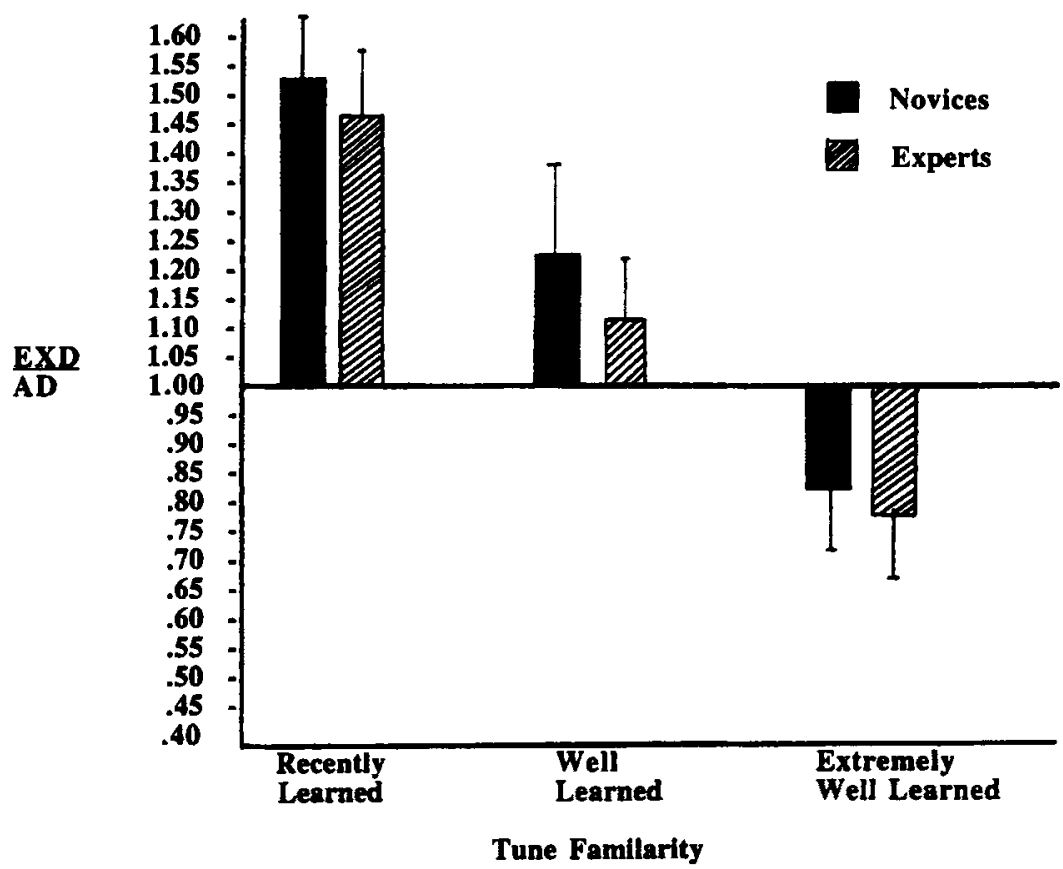

Figure 5. The mean ratio of the subjects' expected duration judgments in Experiment 2 relative to a melody's actual playing time (EXD/AD) as a function of tune familiarity and subjects' degree of musical expertise.

emerged with greater learning experience in which melodies were now underestimated on the order of $15 \%$. These overall differences were confirmed by a set of Tukey post hoc comparisons, with $p=.05$.

Change in preperformance to postperformance duration estimates. When examining the ratio scores in
Figure 5 relative to those of Figure 6, the data suggest that time estimation behavior improved after performing the piano-playing task. In order to assess this observation more directly, a $2 \times 2 \times 3$ ANOVA was conducted that evaluated the signed error of judgments - that is, $[($ judged duration/actual duration $)-1] * 100$ - obtained

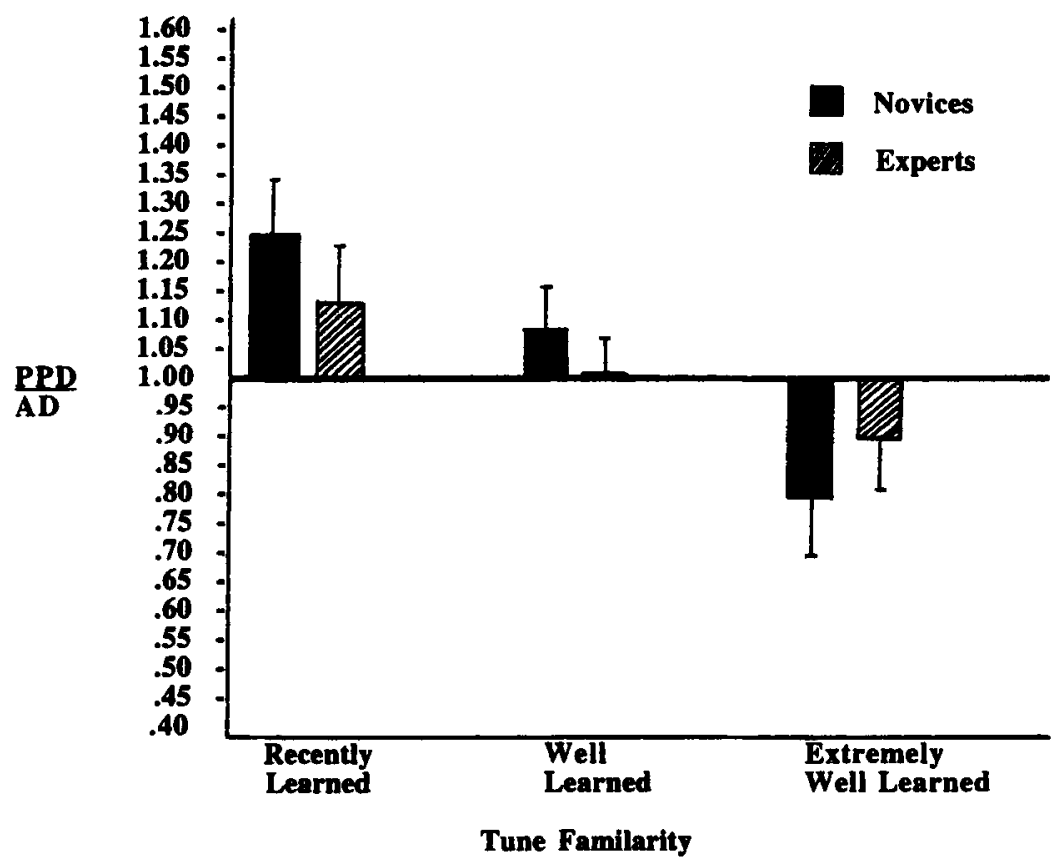

Figure 6. The mean ratio of the subjects' postperformance duration judgments in Experiment 2 relative to a melody's actual playing time (PPD/AD) as a function of tune familiarity and subjects' degree of musical expertise. 


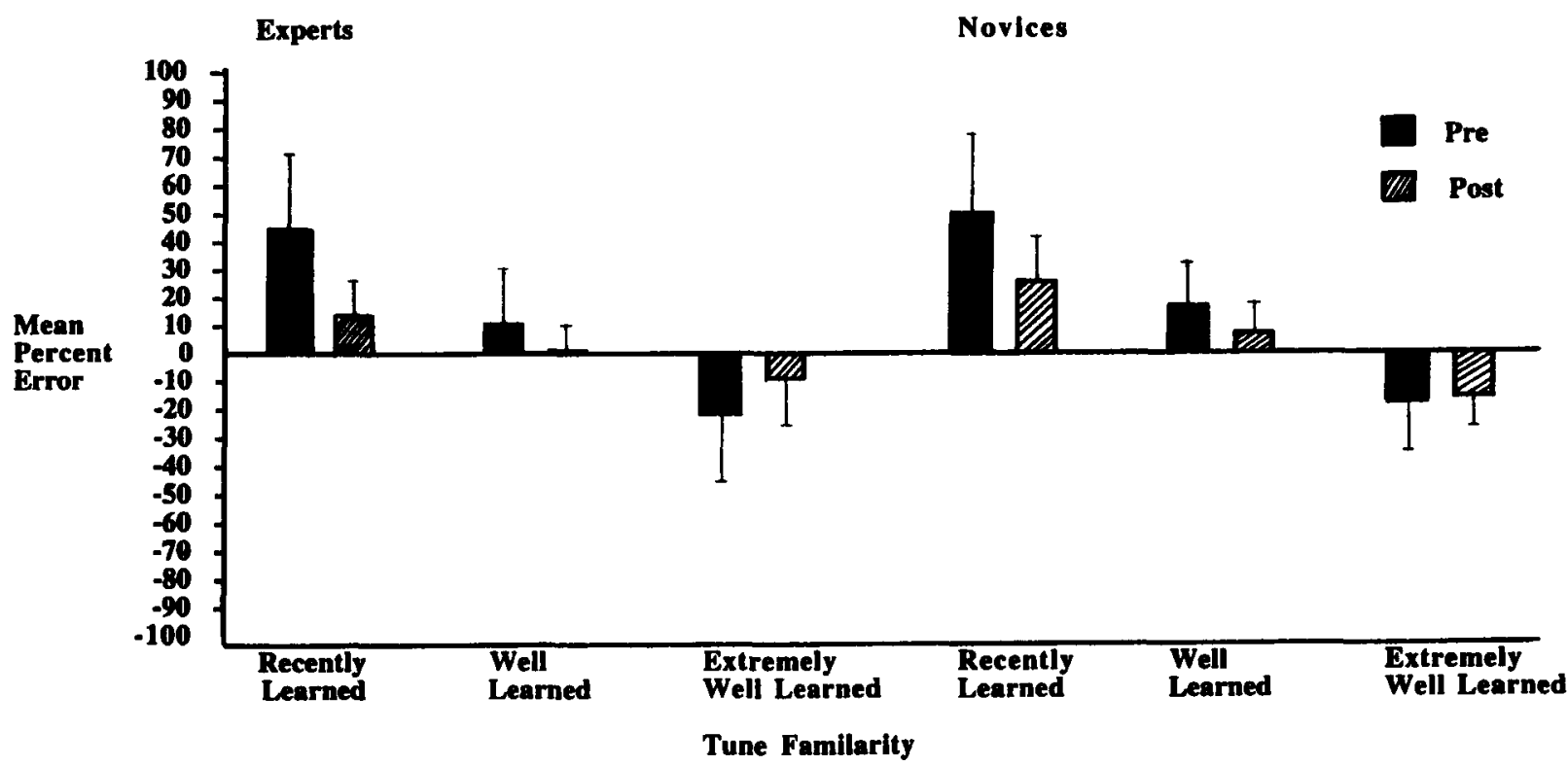

Figure 7. Mean percent signed error in the preperformance and postperformance duration estimates of Experiment 2 as a function of tune familiarity and musical expertise.

both before and after task performance as a function of the two levels of musical expertise and the three levels of tune familiarity.

The most important finding was a three-way interaction $\left[F(2,60)=8.67, M S_{\mathrm{e}}=437, p<.005\right]$, shown in Figure 7. A set of Tukey post hoc comparisons $(p<.05)$ indicates that the experts displayed a significant decline in error from their preestimates (expected) to postestimates of a melody's duration in each of the three learning categories. In fact, these postestimates did not significantly differ from 0 , representing no error at all. The novices, on the other hand, displayed a significant decline in error for only two of the learning categories: the recently learned and well-learned melodies. Melodies that had been extremely well learned did not change from their preduration to postduration estimates and, instead, maintained the same magnitude of underestimation bias. Lastly, in contrast to those of the experts, all of the novices' postestimates significantly varied from a value of 0 .

Covariate analyses. In the first phase of the experimental procedure, two types of ratings were obtained from the subjects that might have potentially influenced time estimation behavior: the judged difficulty of performing a given melody, and a rating of how well the melody was liked. An independent ANOVA on each of these behavioral measures yielded null effects $(F<1)$. Across all three learning categories, both the novices and the experts produced a mean difficulty rating of 5.28 and a mean liking rating of 3.75. In addition, a set of ANCOVAs indicated that duration estimates were not influenced by these two variables.

A third factor was also examined. Recall that, during the experimental procedure, each of the six melodies per- formed by each subject was recorded onto tape. These were subsequently evaluated by two professional musicians (i.e., piano teachers naive to the experiment's purpose), who rated each melody on a 10-point scale for the overall appropriateness of the produced tempo $(10=$ too $f a s t)$ and whether the tempo of the melody's second half perceptually differed from that of the first half $(10=v e r y$ much faster).

To obtain a measure of interrater reliability, each set of ratings from the two judges was correlated with one another to yield $\rho$ s of .92 and .94 , indicating that the judges closely agreed with one another. The ratings themselves were then analyzed through two independent ANOVAs. Judgments concerning any tempo changes within a given composition revealed no significant effects and an overall mean of 4.83. The second set of ratings, however, evaluating the overall appropriateness of a melody's produced tempo, yielded a significant main effect for musical expertise $\left[F(1,30)=5.86, M S_{\mathrm{e}}=1.67, p<.05\right]$. Relative to the experts $(M=5.07)$, the novices tended to play a melody slower $(M=3.87)$ than what was appropriate. In addition, a significant two-way interaction between musical expertise and tune familiarity $[F(2,60)=4.45$, $\left.M S_{\mathrm{e}}=1.21, p<.05\right]$ indicated that this latter effect was primarily confined to early stages of learning. In contrast to the experts who played all three melody types at an appropriate rate, the novices played the recently learned melodies at a slower rate than melodies that were more familiar. Nonetheless, an ANCOVA indicated that these overall ratings did not significantly influence either accuracy or bias of time estimation behavior.

Estimated number of melody segments. One final measure was obtained from each of the six melodies se- 
lected by the subjects. A professional musician was given an unmarked copy of the sheet music from which each melody was performed and was asked to

indicate the boundaries of the melody's natural segments as you would think of them when performing. That is, if you were playing this piece, you may think of it as consisting of different sections. Please mark an $\mathrm{X}$ at the end of each of these sections.

These were then compared with the segmentation scheme provided by the subject, and a ratio score was assigned that evaluated the subject's number of estimated segments relative to the professional's number of estimated segments. Ratios equal to 1 therefore indicate that both individuals identified the same number of segments within a melody, and ratios greater or less than 1 indicate that the subjects identified more or fewer segments, respectively, than the professional.

An overall ANOVA of these data revealed a significant two-way interaction between musical expertise and tune familiarity $\left[F(2,60)=7.18, M S_{\mathrm{e}}=0.86, p<.01\right]$, shown in Figure 8.

For the experts, notice that the ratio of estimated segments in each of the three tune categories did not significantly vary from 1 , indicating that these estimates closely coincided with those of the professional musician. Although the novices displayed a similar pattern of results for both the well-learned and extremely well learned melodies, the number of estimated segments was signif- icantly higher in the category of recently learned melodies $(p<.05)$.

\section{Discussion}

As a set, these overall results converge with those of Experiment 1 and suggest that the effects of learning exert a common influence on different types of remembered duration judgments. Although it is often the case that people estimate an activity's duration in retrospect, it is perhaps just as common to generate an expected duration estimate for an activity that has not yet transpired. These two types of judgments differ in the time lag between the activity's occurrence and the duration estimate itself, because, in contrast to the immediacy of the former, the latter are based on one's collective experience with the activity that has accumulated from various occasions in the past. Nonetheless, the underlying cognitive processes mediating each type of estimate appear very similar in nature. In both cases, an individual must recapitulate the sequence of actions and their respective timing qualities from memory. One would therefore predict a similar influence of learning on both accuracy and bias of judgments, and this in fact is the case.

In Experiment 2, recently learned melodies were not only judged with a high degree of error by both groups of subjects but were also biased toward overestimations from the number of seemingly unrelated chunks or changes in memory (Block, 1985; Ornstein, 1969). Some

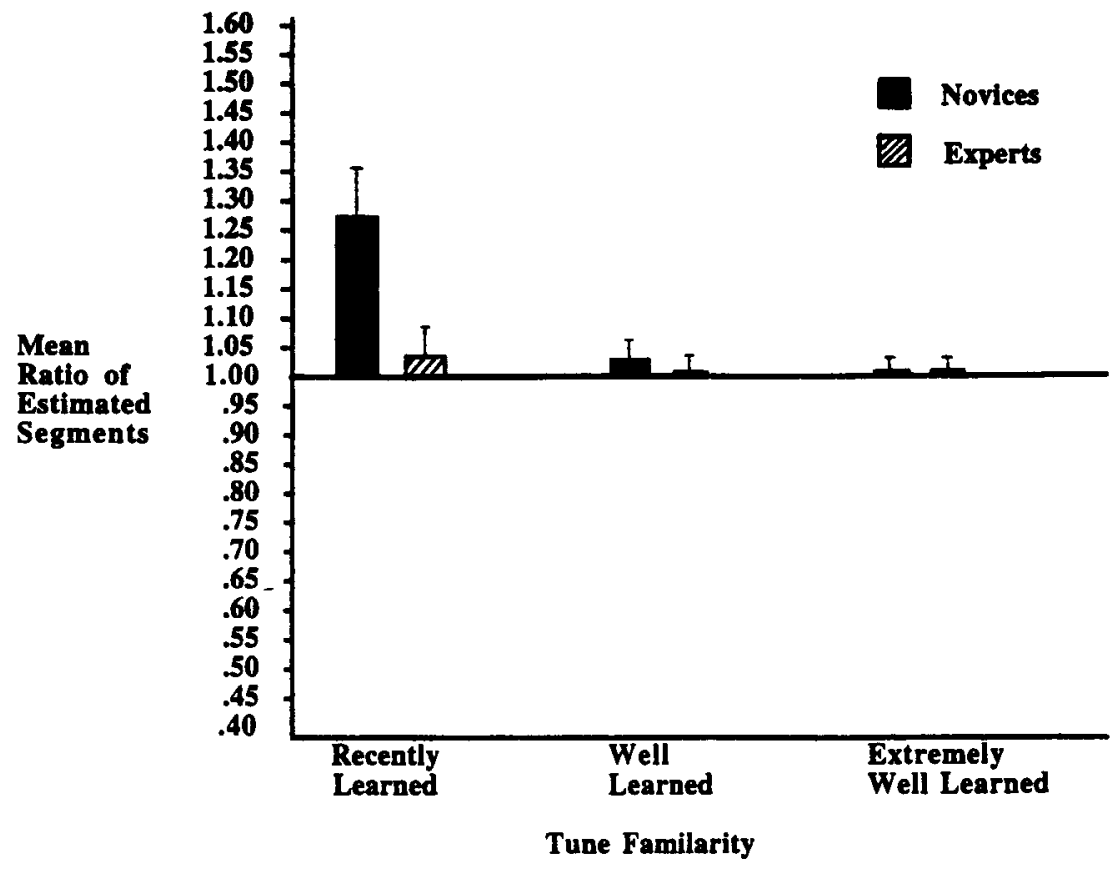

Figure 8. Mean ratio of the number of estimated melody segments (number identified by a subject/number identified by the professional musician) in Experiment 2 as a function of tune familiarity and musical expertise. 
evidence for this idea comes from the segmentation task in which, in contrast to the professional musician, the subjects identified more individual segments in these types of melodies. This difference was a significant one for the novices but only marginally significant for the experts who may have been relying on their more extensive knowledge of music theory to perform the task in lieu of their personal experience with the melodies themselves. Nonetheless, the overestimation bias at early stages of learning was a very robust one that applied to $72 \%$ of all subjects and yielded estimates that, on average, were $48 \%$ longer than the melodies' durations.

As the degree of familiarity increased with welllearned melodies, expected duration judgments became significantly more accurate and, when one examines the frequency distribution of responses, were equally likely to be overestimated or underestimated. Error, however, again increased with melodies that had been extremely well learned. Although $44.5 \%$ of all subjects displayed accurate estimates, $48.5 \%$ significantly underestimated the melodies' durations on the order of $19 \%$. This finding converges with the results of Experiment 1 and more generally has also been observed in the animal literature (see Spetch \& Rusak, 1992, for a review) in which pigeons often respond as if the remembered duration of an event has shortened with time. Two interpretations of this effect have been offered here. One concerns the omission of certain lower level details that may occur in the remembering of highly learned and automaticized events; the second involves the notion of overconfidence. Relative to less familiar activities, extremely well learned ones may seem markedly easier to perform, and, hence, their overall rate of performance may be misremembered as faster than what was actually the case. As before, Experiment 2 did not allow one to determine whether one of these hypotheses was more valid than the other, and it is quite likely that both might have been responsible for the pattern of results. This is an issue that should be addressed in future experiments in which it may be particularly useful to examine the nature of individual differences and why some subjects maintain their high level of accuracy after extensive learning experience but others decline due to underestimations.

The second set of results emerging from Experiment 2 involves the duration estimates obtained after the subjects had performed each individual melody. These types of judgments are more akin to those of Experiment 1, but they cannot be definitively classified as retrospective in nature: Although it is true that the subjects were not instructed that postperformance estimates would later be required, the preceding expected duration task might have led many subjects to monitor time's passage during a melody's performance. Nonetheless, the same pattern of error and bias occurred as a function of learning experience. Unsurprisingly, these estimates were significantly more accurate than those of expected duration, which might be attributed to both the immediacy of the event and the use of conscious time-keeping strategies to facilitate performance. The more interesting finding, however, is the fact that the degree of improvement was more apparent for the experts who produced a high percentage of accurate judgments for each of the three learning categories, but particularly for the well-learned melodies and extremely well learned melodies. In part, this may be due to certain a priori differences between the two subject groups. For example, perhaps people with inherently superior temporal skills are more apt to pursue piano playing as a serious endeavor. A second possibility is that enhanced temporal awareness may be a skill that emerges with the acquisition of expert knowledge in any given field. Even in the absence of direct feedback, experts may be better able to recalibrate their estimates toward greater accuracy after immediately performing the given activity. Again, the results here suggest this is most apt to occur when the material to be judged has not been recently learned but, instead, is quite familiar to individuals.

In sum, the effects of learning observed in Experiment 1 also emerge in a methodological context that examines a different usage of remembered duration than has traditionally been investigated in past research. In addition to confirming that learning influences the error and bias in which an event is judged immediately after its performance, the results indicate that it also plays a role in expected duration judgments that are often used for planning and scheduling purposes. These effects were robust ones that generalized to both novices and experts and to a broad range of compositions that varied between individual subjects. They also cannot be attributed to tempo adjustments. One potential problem in studying expected duration judgments is that subjects may alter the natural rate of their actions so that the total duration of the task is in closer accord with their initial estimatein effect, encouraging a type of self-fulfilling prophecy. Several measures were taken here to minimize this problem: The subjects were not only instructed to play melodies at their natural tempo but were also told that their recorded performances would later be evaluated by a professional judge for the appropriateness of their produced tempi. These precautions appear to have been effective because, when one analyzes the performances themselves, most were produced at the correct rate. The sole exception was the recently learned tunes of the novices, who played these significantly slower than what was appropriate. This, however, did not influence duration estimates, and it cannot account for the pattern of error and bias that was observed as a function of learning experience. Overall, then, the effects observed in Experiments 1 and 2 appear to be quite reliable ones that emerge across different contexts.

\section{GENERAL DISCUSSION}

The results of the present research have implications for several different aspects of time estimation behavior. First, from a theoretical perspective, the constructs of 
learning and event predictability serve to both modify and extend the traditional assumptions of the attentionbased models. According to this latter approach, an event's temporal and nontemporal information are processed by independent mechanisms such that duration estimates will be quite accurate when this dimension is selectively attended to during encoding but highly inaccurate when one primarily attends to nontemporal information. Given that duration is unlikely to be encoded into memory under retrospective situations, people presumably infer duration from the most salient information that is availablenamely, the number of chunks or changes within the nontemporal array.

Evidence for these assumptions was in fact found here when remembered duration was assessed after a relatively few number of learning trials, as has traditionally been done in retrospective research designs. Although this finding can be attributed to independent processing mechanisms, it more generally stems from the lack of structural predictability that accompanies early stages of learning. When an event or activity is relatively unfamiliar, the presence of hesitations and ill-timed movements creates a series of segments that appear temporally disconnected and unrelated to one another. Hence, these types of events will not only be difficult to recapitulate from memory but will result in an independent processing of temporal and nontemporal information, meaning that judged duration will be highly accurate in prospective situations but inaccurate in retrospective ones where the primary focus of attention is on nontemporal information.

With greater learning experience, however, a very different pattern of results emerges. Increased familiarity grants a higher degree of structural predictability in which the individual elements of a time span are now temporally coordinated into a coherent whole. Given that the event's temporal and nontemporal information are lawfully related to one another, both can be jointly encoded into memory with no extra effort: Attending to one dimension will result in the incidental learning of the other (Boltz, 1998a), which in turn results in remembered duration estimates that are quite accurate.

In sum, then, the approach being offered here is one that emphasizes the role of event structure in both attending and remembering, as well as time estimation behavior. Although structural predictability can arise from many different sources, including lower level timing properties (Boltz, 1992, 1994, 1995, 1998a), task knowledge and ordering (Boltz, 1998b), and learning itself, it provides the common function of integrating an event into an organized whole in which the nested subelements appear interrelated to one another. Given that the event's temporal information (i.e., rate, rhythm, duration, and temporal ordering of items) is now lawfully and inextricably entwined with the array of nontemporal information, both structural dimensions can be jointly encoded into memory. These ideas are not necessarily incompatible with the attention-based models but, instead, extend these frameworks by acknowledging the impact of event structure on the way that temporal and nontemporal information are encoded into the cognitive system.

A second feature of the present research is to highlight the use of expected duration judgments as an alternative means in which to investigate remembered duration. Again, these types of estimates are at least as common as retrospective judgments, because duration knowledge is routinely used for planning and scheduling purposes. In the seminal study by Burt and Kemp (1994), the general intent was to simply examine people's ability to predict the total duration of various activities that were not controlled along any particular dimension. Given the broad range of tasks that were used, it is unsurprising that results revealed a high degree of variability in which some activities were judged quite accurately while others were not. The present research extends this earlier work by identifying one factor that influences the overall accuracy of expected duration judgments: an individual's degree of familiarity and past learning experience. However, it should be acknowledged that Experiment 2, in many ways, was preliminary in nature. Although the task of piano playing offered several methodological advantages for the present design, it is also a task that is inherently rhythmic in nature. Hence, additional studies are needed to determine whether the effects of learning generalize to a broader range of activities. In particular, it would be useful to conduct naturalistic studies as a converging operation to laboratory ones in which a measure of task familiarity is treated as a covariate to the activities that are performed. In addition, it would also be useful to investigate the factors, beyond learning experience, that contribute to both the accuracy and the bias of expected duration estimates. Given that the planning and scheduling of activities is such a routine event, this research, in turn, may offer a number of practical applications for everyday behavior.

\section{REFERENCES}

Adams, J. K., \& AdAms, P. A. (1961). Realism of confidence judgments. Psychological Review, 68, 33-45.

BLock, R. A. (1985). Contextual coding in memory: Studies of remembered duration. In J. Michon \& J. Jackson (Eds.), Time, action, and behavior (pp. 169-178). Heidelberg: Springer-Verlag.

BLock, R. A. (1989). Experiencing and remembering time: Affordances, context, and cognition. In I. Levin \& D. Zakay (Eds.), Time and human cognition: A life-span perspective (pp. 333-363). Amsterdam: North-Holland.

Block, R. A., \& ZaKaY, D. (1997). Prospective and retrospective duration judgments: A meta-analytic review. Psychonomic Bulletin \& Review, 4, 184-197.

BoLTz, M. G. (1992). The remembering of auditory event durations. Journal of Experimental Psychology: Learning, Memory, \& Cognition, 18, 938-956.

Boltz, M. G. (1994). Changes in internal tempo and effects on the learning and remembering of event durations. Journal of Experimental Psychology: Learning, Memory. \& Cognition, 20, 11541171

BoLTZ, M. G. (1995). Effects of event structure on retrospective duration judgments. Perception \& Psychophysics, 57, 1080-1096. 
BoLTZ, M. G. (1998a). The processing of temporal and nontemporal information in the remembering of event durations and musical structure. Journal of Experimental Psychology: Human Perception \& Performance, 24, 1087-1104

BolTz, M. G. (1998b). Task predictability and remembered duration. Perception \& Psychophysics, 60, 768-784

Brown, S. W. (1985). Time perception and attention: The effects of prospective versus retrospective paradigms and task demands on perceived duration. Perception \& Psychophysics, 38, 115-124.

BuRT, C. D. B., \& KeMP, S. (1994). Construction of activity duration and time management potential. Applied Cognitive Psychology, 8 155-168.

DE Groor, A. (1966). Perception and memory versus thought: Some old ideas and recent findings. In B. Kleinmuntz (Ed.), Problem solving (pp. 19-50). New York: Wiley.

Egan, D. E., \& Schwartz, B. J. (1979). Chunking in recall of symbolic drawings. Memory \& Cognition, 7, 149-158.

Fraisse, P. (1971). L'apprentissage de l'estimation de la durée et ses repères. Année Psychologie, 71, 371-379.

Fraisse, P., \& OrSINI, F. (1958). Étude expérimentale de conduites temporelles III: Étude génétique de l'estimation de la durée [Experimental study of temporal behavior III: A study of duration estimation]. Année Psychologique, 58, 1-6.

Kahneman, D. (1973). Attention and effort. Englewood Cliffs, NJ: Prentice-Hall.

LoEHLIN, J. C. (1959). The influence of different activities on the apparent length of time. Psychological Monographs, 73, (4, Whole No. 474).

Miller, G. W., Hicks, R. E., \& Willette, M. (1978). Effects of concurrent verbal rehearsal and temporal set upon judgments of temporal duration. Acta Psychologica, 42, 173-179.
MoHan, V. (1975). The influence of stimulus modality, duration, and knowledge of results on improvement in temporal estimation. Manas, 22, 159-167.

MONTARE, A. (1985). Learning effects of knowledge of results upon time estimation. Perceptual \& Motor Skills, 60, 871-877.

MonTARE, A. (1988). Further learning effects of knowledge of results upon time estimation. Perceptual \& Motor Skills, 66, 579-588.

NAVON, D., \& GOPHER, D. (1979). On the economy of the humanprocessing system. Psychological Review, 86, 214-255.

ORNSTEIN, R. (1969). On the experience of time. Baltimore, MD: Penguin.

OSKAMP, S. (1962). The relationship of clinical experience and training methods to several criteria of clinical prediction. Psychological Monographs, 76(28, Whole No. 547).

POYNTER, W. D. (1983). Duration judgment and the segmentation of experience. Memory \& Cognition, 11, 77-82.

SPETCH, M. L., \& RusaK, B. (1992). Time present and time past. In W. K. Honig \& J. G. Fetterman (Eds.), Cognitive aspects of stimulus control (pp. 47-67). Hillsdale, NJ: Erlbaum.

ZAKAY, D. (1989). Subjective time and attentional resource allocation: An integrated model of time estimation. In I. Levin \& D. Zakay (Eds.), Time and human cognition (pp. 365-397). Amsterdam: Elsevier.

ZAKAY, D., \& MERAN, V. (1988). A meta-analysis of time estimation research. Unpublished manuscript, Tel Aviv University.

Zakay, D., Tsal, Y., Moses, M., \& Shahar, I. (1994). The role of segmentation in prospective and retrospective time estimation processes. Memory \& Cognition, 22, 344-351.

(Manuscript received June 2, 1997; revision accepted for publication February 17, 1998.) 